\title{
LA DIOSA ATAECINA Y EL NOMBRE DE LA NOCHE EN ANTIGUO IRLANDÉS
}

\author{
Eugenio RAMÓn LujÁn MARTíneZ
}

In the last years the number of known inscriptions devoted to the Hispanic goddess Ataecina has greatly increased due to new findings. They have provided us with some new variations of her name, which can serve as the basis for a reconsideration of the etymologies so far proposed for it. A conexion with Old Irish adaig 'night' has sometimes been advocated for, which after analysing the whole evidence now available proves to be right. Nevertheless, the etymology of that Irish word was itself in need of revision. Both seem to have been built on *adak-, analysable as the prefix ad-followed by the IE root *ak-. We would thus have here an innovation common to an Hispano-Celtic dialect and Goidelic.

\section{Introducción ${ }^{1}$}

La etimología del teónimo hispano Ataecina, que conocemos únicamente en inscripciones latinas de la península Ibérica, es discutida. De hecho, las opiniones de los estudiosos que se han ocupado del tema están divididas en dos grupos: por un lado se encuentra la de aquéllos que, siguiendo la propuesta implícitamente planteada por D'Arbois de Juvainville (1893, p. 389$)^{2}$, sostienen que se trata de un compuesto *ate-gina que vendría a

1 Este artículo ha sido realizado dentro del proyecto de investigación PB96-0615, cuyo investigador principal es el Prof. Javier de Hoz, a quien agradezco las observaciones que me ha hecho sobre una versión anterior de este trabajo.

2 «Le nom de la déesse Ataecina ou Adaegina peut être corrigé en *Ate-gena qui serait un mot gaulois.» 
significar, por tanto, la «Renacida» ${ }^{3}$; por otro, hay quienes como Steuding (1884-86), Balmori (1932), Albertos (1966, p. 38) o Untermann (1987, p. 66) defienden ${ }^{4}$ que hay que relacionar dicho teónimo con a. irl. adaig 'noche', palabra que en esa lengua ha acabado por sustituir al viejo térmi-

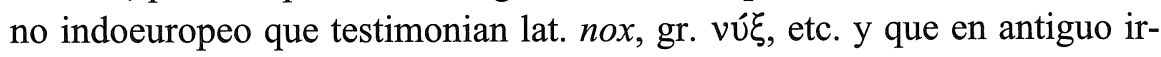
landés sólo ha sobrevivido en la expresión adverbial $i$ n-nocht 'esta noche' $\mathrm{y}$ tal vez en un par de compuestos ${ }^{5}$.

No podemos basarnos en datos externos para inclinarnos a defender una u otra etimología. Ataecina aparece identificada en una conocida inscripción de Mérida (CIL II 462) con Proserpina, a la que ahora se une un epígrafe todavía inédito de Salvatierra de los Barros BA ${ }^{6}$. De tal identificación ${ }^{7}$ se ha intentado inferir tanto su carácter agrario ${ }^{8}$, como - más habitualmente - su carácter infernal ${ }^{9}$, idea que pudiera verse reforzada en el caso de que precisamente el culto a esta diosa hubiera sido un factor favorecedor de la difusión de cultos mistéricos a Cibeles en el occidente hispano, como a veces se ha propuesto ${ }^{10}$. Pero en cualquier caso $-\mathrm{y}$ esto es lo que ahora nos interesa - tanto una como otra etimología son compatibles con estos datos histórico-arqueológicos ${ }^{11}$.

3 Así, Leite de Vasconcelos 1905, pp. 161-163, Schmidt 1957, p. 136, Evans 1967, p. 144. Con todo, D’Arbois 1906, p. 106, años más tarde habría de afirmar que la etimología del teónimo era desconocida.

4 Escéptico frente a esta relación entre adaig y Ataecina, Evans 1993, p. 597, aunque no ofrece argumentos.

5 V. DIL, s.u. nocht.

6 V. Abascal 1995, p. 89. Conozco el texto íntegro de la inscripción gracias a la amabilidad del Prof. J. L. Ramírez Sádaba.

7 Tal vez también atestiguada en otro epígrafe de las cercanías de Mérida (CIL II 461) que presenta la secuencia $D S A T P$. Esta última letra fue interpretada por Leite 1905 , p. 150 , como $P$ (roserpinae). La interpretación no es segura, pero, habida cuenta de la fraseología presente en la inscripción de Mérida, en la que la mención Proserpina sigue a la secuencia habitual de advocaciones de la diosa, no es en absoluto descabellada.

8 Así Leite 1905, pp. 163-164.

9 V., p. ej., Blázquez 1962, pp. 145-146, y 1975, s.u.

10 V., p. ej., García y Bellido 1967, p. 44, Bendala 1981, p. 289.

11 El vegetal que aparece como decoración de un cipo consagrado a una Dee Sancte no puede considerarse como un elemento decisivo para atribuir a la diosa un carácter agrario, como quería Leite 1905 , pp. 174-175, pues, aparte de que no parece que se puedan relacionar con el culto de Ataecina todas las inscripciones con la mención Dea Sancta (v. ahora Abascal 1995, pp. 80-86), dicho vegetal ha sido interpretado posteriormente como un ciprés, lo que, unido al carácter funerario del cipo en sí, parecería reforzar la interpretación opuesta (Blázquez 1962, pp. 145-146, y 1975, s.u.). 
La aparición en los últimos años de un buen número de epígrafes consagrados a esta divinidad, especialmente en la ermita de Santa Lucía del Trampal (Alcuéscar CC) - lugar en el que ahora hay acuerdo en aceptar que debió existir en la antigüedad un santuario consagrado a esta divini$\mathrm{dad}^{12}$ - nos ha proporcionado nuevas e interesantes variantes del nombre, por lo que parece el momento de replantear la cuestión de su etimología, que desde el artículo de Balmori 1932, aparecido en estas mismas páginas de EMERITA, no había vuelto a recibir tratamiento detallado.

Comenzaremos por ofrecer todas las variantes atestiguadas actualmente del nombre de la diosa, para ocuparnos seguidamente de la etimología de a.irl. adaig, que también será conveniente replantear, pues el tratamiento de Vendryès en su diccionario etimológico del irlandés y el que se encuentra en otros estudios de principios de siglo hoy ya no parecen sostenibles en los términos en que fueron formulados. En relación con esto veremos las posibilidades de interpretación del nombre de la divinidad hispana.

\section{El nombre de la diosa}

Ofrecemos a continuación las variantes conocidas del nombre de la divinidad con referencia al lugar de hallazgo de la inscripción en que se documentan y a su edición ${ }^{13}$.

Adecine (dat.): Alcuéscar (Abascal 1995, n. ${ }^{\circ}$ 1).

Adaecinae (dat.): Alcuéscar (Abascal 1995, n. ${ }^{\circ}$ ).

Adaeginae (dat.): Medellín (CIL II 605; Leite 1905, p. 148; Blázquez 1962, p. 142; López Melero 1986, p. 108).

[A]deginae (dat.): Alcuéscar (Abascal 1995, n. ${ }^{\circ}$ 9).

Adegine (dat.): Alcuéscar (Abascal 1995; n. ${ }^{\circ}$ 6).

Addaecin(ae) (dat.): Alcuéscar (Abascal 1995, n. ${ }^{\circ} 10$ ).

Add[-]ina[e?] (dat.?): Salvatierra de Santiago (Roso de Luna 1904, n. ${ }^{\circ} 10$; Hurtado de San Antonio, n. ${ }^{\circ}$ 432; Madruga y Salas 1995, pp. 335-338; Abascal 1995, p. 88).

Ataecina (nom.): Mérida (CIL II 462; Leite 1905, pp. 146-147; Blázquez 1962, p. 141; García Iglesias 1972-73, n. ${ }^{\circ}$ 12; López Melero 1986, p. 107).

12 V. García-Bellido 1991, pp. 69-71, y Abascal 1995, p. 94.

13 A las inscripciones aquí mencionadas habría que añadir una procedente de El Gordo (Cáceres), citada por de la Vega 1992, p. 336, todavía inédita y cuyo texto desconocemos. 
Ataecinae (dat.): Mérida (Ephemeris Epigraphica IX 42; Leite 1905, pp. 147-148; Blázquez 1962, pp. 141-142; García Iglesias 1972-73, n. ${ }^{\circ}$ 13; López Melero 1986, p. 107), Caleruela (Cortés et alii 1982, pp. 74-75; HEp.1, n. ${ }^{\circ}$ 602; $A E$ 1986, n..$^{\circ} 426$; González-Conde 1988, p. 131; HEp.2, n. ${ }^{\circ} 679$ ), Talavera de la Reina (Urbina 1993-94, pp. 29-30).

Attaec[i]nae (dat.): Alcuéscar (Abascal 1995, n. ${ }^{\circ}$ 8).

[A]ttaegina(e) (dat.?): Bienvenida (Esteban Ortega 1984; López Melero 1986). Atteginae (dat.): Alcuéscar (Abascal 1995, n. ${ }^{\circ} 3$ ).

A[-]ecin[-]: Alcuéscar (Abascal 1995, n. $\left.{ }^{\circ} 14\right)$.

[A-]ae[-]ina(e) (dat.?): Santa Ana ${ }^{14}$ (Blázquez 1962, pp. 143-144; López Melero 1986, p. 108).

[A-]aegin[-]: Salvatierra de Santiago (Madruga y Salas 1995, pp. 331-335).

Nombre abreviado en $A$ : Alcuéscar (Abascal 1995, n. ${ }^{\circ}$ 5), Cárdenas (CIL II 461; Leite 1905, p. 150; Blázquez 1962, p. 143; García Iglesias 1972-73, n. ${ }^{\circ} 11$; López Melero 1986, p. 108), Malpartida de Cáceres (CIL II 5299; Leite 1905, p. 149; Blázquez 1962, pp. 142-143; Hurtado 1977, n. ${ }^{\circ} 332$; López Melero 1986, p. 110), Ibahernando (Ephemeris Epigraphica IX 101; Leite 1905, pp. 150-151; Blázquez 1962, p. 143; Hurtado 1977, n. 294; López Melero 1986, p. 109), Cabeza del Griego ${ }^{15}$ (CIL II 5877; Blázquez 1962, p. 144), Cagliari ${ }^{16}$ (CIL X 7557; Blázquez 1962, p. 144).

Nombre abreviado en $A D$ : Alcuéscar (Abascal 1995, n. ${ }^{\circ} 13$ ), Malpartida de Cáceres (CIL II 5298; Leite 1905, p. 149; Blázquez 1962, p. 142; Hurtado 1977, n. ${ }^{\circ} 331$; López Melero 1986, p. 110).

Nombre abreviado en ATE: Salvatierra de los Barros (Abascal 1995, p. 89).

Sin mención del teónimo: Alcuéscar (Abascal 1995, n. ${ }^{\circ}$ 2), Alcuéscar (Abascal 1995, n. ${ }^{\circ}$ 7), Alcuéscar (Abascal 1995, n. ${ }^{\circ} 11$ ), Alcuéscar (Abascal 1995, n. ${ }^{\circ}$ 15), Mérida (Ephemeris Epigraphica IX 43; Leite 1905, pp. 149-150; Blázquez 1962, pp. 142-143; García Iglesias 1972-73, n. ${ }^{\circ}$ 15; López Melero 1986, p. 108), Mérida (HAE 2681; García Iglesias 1972-73, n. ${ }^{\circ}$ 14), Mérida (HEp.2, n. ${ }^{\circ} 34$ ), Herguijuela (Callejo 1970, p. 144; Hurtado 1977, n. ${ }^{\circ} 263$; López Melero 1986, p. 109), Herguijuela (Callejo 1970, p. 146; Hurtado 1977, n. ${ }^{\circ} 260$; López Melero 1986, pp. 109-110), Quintos? (CIL II 71; Leite 1905, pp. 151-154; Blázquez 1962, p. 143; Encarnaçâo 1975, p. 116; Encarnaçâo 1984, n. ${ }^{\circ}$ 287; López Melero 1986, p. 110), Quintos (CIL II 101; Leite 1905, p. 151; Blázquez 1962, p. 143; Encarnaçâo 1984, n. 288; López Melero 1986, p. 111).

14 Tradicionalmente se citaba esta inscripción como procedente de Ibahernando, cuando en realidad procede de Santa Ana (Abascal 1995, p. 89, n. 237).

15 Actualmente hay un cierto escepticismo en cuanto a que esta inscripción proceda realmente de Segóbriga. V. Abascal 1995, p. 91, n. 257, según indicaciones de M. Almagro Gorbea.

16 En realidad, no parece que proceda exactamente de Cagliari, sino de un balneario próximo (Zucca, e. p.). 
A partir de un primer análisis de las variantes del nombre podemos asumir de manera incontrovertible los hechos siguientes:

a) La secuencia final del teónimo es indiscutiblemente -ina, pues esta parte permanece invariable en todos los testimonios del nombre de la diosa ${ }^{17}$.

b) El teónimo empieza por $A$-, pues tampoco aquí hay variaciones.

c) La primera consonante del teónimo es una dental, que aparece como $-d$-, $-d d-,-t$ - y -tt-, por lo que aquí se plantea el problema de cuál es la consonante etimológica (sorda o sonora y geminada o simple). En cualquiera de las cuatro soluciones posibles habría que explicar, además de la etimología, a qué se debe el ensordecimiento o la sonorización y la geminación o simplificación de la geminada.

d) En la segunda sílaba alternan la vocal -e- y el diptongo -ae-. También aquí habría que justificar, además de la etimología, bien la monoptongación, bien el surgimiento del diptongo si es que éste resultara ser secundario.

e) Para la segunda consonante del teónimo alternan $-c-$ y - $g$-, lo que plantea problemas similares a los aludidos en los dos puntos anteriores.

Éstos son los datos objetivos a los cuales ahora habría que dar interpretación, pero postponemos el intento hasta haber tratado de la etimología de a.irl. adaig 'noche'.

\section{La etimología de a.irl. adaig 'noche'}

La etimología de a.irl. adaig (gen. aidche) 'noche' presenta enormes complicaciones. Vendryès, en su diccionario etimológico (LEIA, s.u.), da muestras de buen juicio al comenzar el tratamiento de la misma con la constatación de que carece de etimología segura. Repasaremos brevemente las propuestas de Stokes y de Pedersen, que nos servirán de punto de partida para nuestras propias reflexiones ${ }^{18}$.

17 Con lo que queda invalidada ya de entrada la etimología de d'Arbois, pues, a pesar de que, contra la opinión de Balmori 1935, sí que tenemos -ginos por -genos en la península Ibérica (v. Albertos 1966, pp. 279-280), el corpus de inscripciones consagradas a Ataecina es ya demasiado amplio como para asumir que no se nos ha transmitido ninguna variante -gena por mera casualidad.

18 Dejo de lado la etimología propuesta por Thurneysen 1927, p. 184, n. 2, para quien adaig habría tenido originariamente el sentido de 'intervalo, periodo de tiempo', pues, como ya señaló Pedersen 1933, pp. 71-72, se debe a una mala interpretación de adaigh en un pasaje de las leyes (Laws V 482 = Binchy 1978, p. 1872, lín. 8), donde es una variante ortográfica del ac.-dat. athaig, que está por el nom. athach, palabra ésta que sí que tiene el significado atribuido por Thurneysen a adaig. 
Para Stokes (1894, p. 326), se trataría de un originario compuesto *ad-ak $k^{w} \bar{l}$, cuyo segundo elemento sería relacionable con el lat. aquilus 'castaño oscuro' y con opācus 'oscuro'. Pedersen (1933, p. 72) calificó esta explicación de «evidentemente errónea» y Vendryès (LEIA, s.u.) la tacha de fantasiosa, pero ninguno de ellos ofrece argumentos.

Por su parte, Pedersen (1933) dedicó un artículo a la relación entre adaig y lit. júodas 'negro', para lo cual explicaba adaig a partir de una proto-forma * $(j) a d a k \bar{~} \mathrm{y}$, además, se veía obligado a admitir que la primera $a$ procedía de un schwa que alternaba con $\bar{o}$. Esta forma con $\bar{o}$ sería la que explicaría el adjetivo lituano. Naturalmente, de la yod inicial que presenta la palabra lituana no hay ningún resto en antiguo irlandés, pero Pedersen, además, postula ad hoc la aparición de prótesis ante $\bar{o}$ - en indoeuropeo. Y va todavía más allá al sugerir un posible parentesco entre esas dos palabras y lat. àter 'negro', a la que hace remontar a *ādro-. Queda patente que su propuesta no pasa de ser un mero cúmulo de hipótesis sin ninguna verosimilitud.

Adaig pertenece a un pequeño grupo de palabras femeninas del antiguo irlandés, que, como señaló por primera vez Thurneysen (1887, pp. 145-147) ${ }^{19}$, se caracterizan por presentar un nominativo singular de tema en $-\bar{\imath}$ que se opone al resto de la declinación del singular como tema en $-y \bar{a}$. Sin embargo, entre estos temas no hay ninguno que constituya un buen paralelo para adaig, en cuya primera consonante alterna el carácter palatal y no palatal a lo largo del paradigma. Las otras palabras de esta clase son monosílabos en el nominativo (como méit o caill) o palabras cuya segunda sílaba en el nominativo empieza bien por consonante palatal que se mantiene como tal a lo largo de toda la declinación (como sétig, inis, Brigit, etc.), bien por consonante no palatal igualmente mantenida a lo largo de toda la declinación (p. ej., fochraicc).

En cualquier caso, resulta evidente que hay que asumir para establecer la etimología de adaig, gen. aidche, una secuencia *ad $\mathrm{V} k \bar{l}-\mathrm{o} *^{*} a d \mathrm{Vk}^{w} \overline{-}-$ $\left(\mathrm{V}=\right.$ vocal) para el nominativo ${ }^{20}$. En cuanto a la vocal de la segunda síla-

19 V. también las listas complementarias de Stokes 1887, pp. 289-291, así como las explicaciones de las gramáticas de Pedersen 1913, pp. 88-89, y Thurneysen 1946, pp. 184-187.

20 Cf. Michelena 1961, p. 202: «Piénsese lo que se piense del nombre Ataecina y de sus variantes [...], lo que es seguro es que el irl. ant. adaig 'noche' supone $d$ (acaso el prefijo $a d-)$ y *ko *q.» 
ba, parece descartable que pudiera ser una -i-, pues en tal caso también esperaríamos una calidad palatal en la $-d$ - del nominativo. Lo único que el análisis interno de esta palabra en antiguo irlandés nos permite deducir directamente es precisamente que la vocal de la segunda sílaba no producía palatalización: el caracter palatal de la $-d$ - en los casos diferentes del nominativo se debería a que, tras la síncopa regular de la vocal de la segunda sílaba en los casos de la declinación en que tras la reducción de las sílabas finales la palabra aún tenía más de dos sílabas, se habría producido una asimilación de palatalidad en el grupo consonántico originado secundariamente ${ }^{21}$.

\section{Ataecina y a.irl. adaig}

Retomando la cuestión donde la dejábamos planteada más arriba, los problemas que surgen a propósito del teónimo Ataecina son relacionables fundamentalmente con la evolución del latín de España. Las dudas que se plantean son, pues, en cuanto a las consonantes, si las grafías con oclusiva sorda $(t \mathrm{y} c)$ son las etimológicas y las grafías con sonora se deben a un banal proceso de sonorización ${ }^{22}$, como creía Tovar (1949, p. 140), o bien las grafías con sonora son las etimológicas y las grafías con sorda se deben a un fenómeno de hipercorrección, del que hay abundantes paralelos en la antroponimia del área vetona ${ }^{23}$ : Botilla (Pedroso de Acim CC) y Boutius (Barrado CC, Garrovillas CC, Miajadas CC, Candeleda AV, Hinojosa de Duero SA, etc.) frente a los nombres en Boud- (de *bhoudh(i)-

21 V. Lewis y Pedersen 1989, p. 100 y Greene 1973, p. 135, frente a la opinión de Thurneysen 1946, pp. 98-99, para quien la regla general es que los grupos consonánticos surgidos de la síncopa cuya primera consonante era originariamente neutra y la segunda, palatal, sufren despalatalización.

22 Con independencia de que éste pueda en último término ponerse en relación o no con la lenición céltica. De hecho, curiosamente, la «lenición» que se atestigua en los antropónimos del territorio vetón se corresponde más con el tipo britónico que con el gaélico (v. Luján, e. p.).

23 La dispersión de los epígrafes de Ataecina conocidos hoy en día permite suponer con buen fundamento que esta divinidad era fundamentalmente vetona (Abascal 1995, pp. 103-105). Para los límites de los vetones pueden seguirse los trabajos de Roldán 1968-69, pp. 101-106; Salinas de Frías 1986, pp. 21-22; González-Conde 1986 y Sayas y López Melero 1991, pp. 79-80, pero teniendo en cuenta las observaciones críticas de Sánchez Moreno 1995, pp. 486-487. 
'victoria'), Epurus (Puerto de Santa Cruz CC) por Eburus, Malcenus (Ibahernando CC) frente a Malgeinus, Matucenus (Valdelacasa de Tajo CC, Ávila) por Matugenus, etc.

En cuanto al vocalismo de la segunda sílaba, hay que plantearse también si $e$ es meramente el resultado de la evolución de $a e$ o bien $a e$ es una grafía hipercorrecta por $e$. En el primer caso, hay que considerar, además, la posibilidad de que ae sea etimológico o bien se deba a un fenómeno de «infección» céltica, como se ha defendido habitualmente (p. ej., Albertos 1966, p. 38) a partir de la constatación de la existencia del antropónimo Atacina en Ampurias (CIL II $4627=$ Almagro 1952, n. $\left.{ }^{\circ} 14\right)^{24}$. También de este fenómeno contamos con un buen número de paralelos en la onomástica del territorio vetón: Aisus (Hinojosa del Duero SA), Sailgius (Abertura CC, Casas de Millán)/Saelgius (Martiago SA), Maicira (Ávila), Calaetus (Torrejón el Rubio CC), Balaesus (Trujillo CC, Talavera la Vieja CC), Vacaemus (Ávila), etc. ${ }^{25}$

Así pues, para intentar dar respuesta a los diferentes interrogantes que planteábamos en $\S$ II y poder así dilucidar si es posible o no relacionar el teónimo hispano con la palabra irlandesa, resulta interesante explorar una vía de análisis interno de los documentos epigráficos, hasta el momento no utilizada, que nos permita contextualizar los datos lingüísticos que ofrece el teónimo por sí solo. De hecho, los epígrafes relevantes son los siguientes:

A) Alcuéscar (Abascal 1995, n. ${ }^{\circ}$ 1): Adecin/e Sanctle Viuial Patric/ia A(nimo) L(ibens) V(otum) S(oluit).

Como se observa ${ }^{26}$, la aparición de $e$ en la segunda sílaba del teónimo se correlaciona con la monoptongación de la desinencia de dativo del propio teónimo y del adjetivo sancte.

B) Alcuéscar (Abascal 1995, n. ${ }^{\circ}$ 6): Do(minae) D(eae) S(anctae)/ Turib/ri(g)e(nsi) Aldegin/e Iulia/ [S]euer(a)/ [e]x Vot(o)/L(ibens) A(nimo) S(oluit).

24 Ho. I 252-253, en cambio, deriva Atacina del nombre Atax, que entraría en la bien conocida serie de antropónimos en $A t(t)$-.

25 V. Luján, e. p.

26 En Abascal 1995, p. 34, hay una inconsecuencia entre la edición de la inscripción y el comentario, pues en la edición aparece Adecin[a]/e Sanct-/ae cuando en el comentario se nos dice que en las dos primeras líneas está clara la monoptongación de -ae en -e. La fotografía que acompaña al texto permite dilucidar la cuestión a favor de la lectura que presupone el comentario frente a la que se da en la edición. 
Nuevamente ${ }^{27}$ hay aparición de $e$ en la segunda sílaba y monoptongación de la desinencia de dativo. Creo que la -e de Turibrie también es un dato a tener en cuenta, pues a pesar de que Abascal restituye Turibri(g)e(nsi), continuando con la práctica habitual para otros epígrafes dedicados a esta divinidad, Turibrie puede ser perfectamente un genitivo del nombre de la ciudad Turibriga, simplemente con pérdida de -g-intervocálica.

De hecho - y me permito aquí realizar un excurso acerca de esta cuestión - en ni uno solo de los epígrafes de Ataecina conocidos hasta el momento tenemos atestiguado el adjetivo en -ensis referido a la diosa, pues las restituciones habituales son arbitrarias. En Mérida (CIL II 462) aparece la secuencia Dea Ataecina Turibrig y también (Ephemeris Epigraphica IX 43) deae sanc Turib; en Medellín (CIL II 605) dominae Turibrig Adaeginae; en Herguijuela (López Melero 1986, p. 109) D[[a]]eae Sanctae Turibrige; en Alcuéscar D D Turibri Atteginae (Abascal 1995, n. ${ }^{\circ}$ 3), D D $S$ Turibri Adaecinae (Abascal 1995, n. ${ }^{\circ}$ 4), Domina[e] Turibr Attaec[i]nae (Abascal 1995, n. ${ }^{\circ}$ 8), etc., y en el epígrafe conservado en Cabeza del Griego (CIL II 5877) Dominae S Tur. En todas ellas, además de en las que únicamente presentan una $T$ como abreviatura del topónimo (así las de Cárdenas BA, CIL II 461; Malpartida de Cáceres CC, CIL II 5298; etc.), es habitual suponer una forma del adjetivo Turi-/Turo-/Turubrigensis, que, como queda patente, no tenemos de hecho atestiguado de forma inequívoca en referencia a la diosa. En cambio, un genitivo Turi-/Turo-/Turubrigae resultaría aceptable en ellas ${ }^{28} \mathrm{y}$, además, sí que lo tenemos bien atestiguado en la forma Turobrigae de una inscripción de Mérida (Ephemeris Epigraphica IX 42), a la que hay que añadir Turubriga(e) de la de Bienvenida (López Melero 1986). Alternativamente, se podría pensar, según me sugiere el Prof. Javier de Hoz, que Turi-/Turo-/Turubriga fuera un adjetivo en - $a$ formado a partir de *Turibrix y mantenido sin latinizar en -ensis por ser un epíteto ritual de la diosa.

27 Abascal desarrolla Adegin(a)e, restituyéndo el dativo clásico.

28 De hecho, en la inscripción de Herguijuela, no es que sea aceptable, sino que es lo que realmente aparece, pues Turibrige es el genitivo con evolución $a e>e$ del diptongo final. De los problemas que la diferenciación entre ae y e etimológicas causaban al grabador es buena muestra el daeae inicial en la misma inscripción, que debe indicar una pronunciación /dee/. 
Por otra parte, - y volviendo ya al análisis de este epígrafe concretola desaparición de la - $g$ - intervocálica de Turibrie, con independencia de que se trate del adjetivo gentilicio o del genitivo del topónimo, es un dato muy interesante si lo comparamos con Adegine, pues de ser la - $g$ - originaria en el teónimo se esperaría el mismo tratamiento; no, en cambio, si en realidad procede de $*-k-{ }^{29}$.

C) Alcuéscar (Abascal 1995, n. 9): Dominael Turibri(gensi)/ [A]deginae/ [-] Caesius/ Cresces/ L(ibens) A(nimo) V(otum) S(oluit)

Esta inscripción, en cambio, presenta un testimonio ambiguo, ya que, por un lado, tenemos -e- en la segunda sílaba del diptongo y, por otro, aparece el diptongo ae etimológico tanto en las desinencias de dativo femenino como en el nombre Caesius.

D) Mérida (CIL II 462): Dea . Ataecina Turi/brig(ensis) Proserpinal per tuam maiestatem/ te rogo oro obsecro/ uti uindices quot mihi/ furti factum est quisquel mihi imudauit inuolauit/ minusue fecit eas q(uae) i(nfra) s(criptae) $s(u n t) /$ tunicas VI [p]aenula/ lintea II in[dus]ium cu/ius I G V [...] ignoro/ A [......] ius/ui

En esta inscripción, donde encontramos una grafía -t- para la primera consonante del teónimo, podemos constatar, sin embargo, que la sonorización de las oclusivas sordas intervocálicas ya se había producido, según muestra la forma verbal imudauit, lo que invita a interpretar la -t- de Ataecina como una grafía hipercorrecta. En cuanto a los diptongos, junto a Ataecina tenemos [p]aenula. Esta palabra habitualmente es considerada un hápax, pero si la $p$ - inicial está bien restituida - y en mi opinión sí lo está a juzgar por el contexto de términos relativos a la ropa en que se inserta-, podría tratarse simplemente de una forma de pannulus, diminutivo de pannus que tenemos atestiguado en fuentes literarias ${ }^{30}$. El diptongo -ae- podría deberse a infección vocálica provocada por la - $u$ - de la sílaba siguiente, para lo que contamos con un posible paralelo en el antropónimo

29 Significativamente, en el conjunto de los antropónimos atestiguados en territorio vetón, todos los ejemplos de desaparición de oclusiva sonora intervocálica ocurren con -g-: Catuenus (Malpartida de Cáceres CC) por *Catugenus, Mailo (Torrequemada CC) por Magilo, Maila (Malpartida de Cáceres CC) por *Magila, Matueniqum (Yecla de Yeltes, SA) por Matugenicum y Meduenus (Plasenzuela CC)/Meiduenus (Malpartida de Plasencia CC, Nuñomoral CC, Abertura CC) por Medugenus (v. Luján, e. p.).

30 V. $O L D$, s.u. 
Meiduenus (frente a Meduenus; ambos de *Medugenos), atestiguado en varias inscripciones de territorio vetón (Malpartida de Plasencia $\mathrm{CC}, \mathrm{Nu}-$ ñomoral CC, Abertura CC).

E) Caleruela (González-Conde 1988): Bassus/ Turobri(gensis)/ eques a/lae uetto/num ara/m pos(su)it/ Ataecin/ae uotu/m solui(t)/ [l]iben[s]/ [merito]

De nuevo, conservación del diptongo ae en todos los casos. Frente al ejemplo anterior, aquí la -t- intervocálica está conservada en uotum y, en cambio, tenemos Ataecinae.

F) Medellín (CIL II 605): Dominae /Turibrig(ensi) /Adaeginae /Maritum

Un ejemplo más de conservación de los dativos finales en -ae al mismo tiempo que aparece Adaeg-. Además tenemos conservada la - $t$ - intervocálica en Maritum y, en cambio, la primera oclusiva del teónimo aparece notada como - $d$-, lo que parece apuntar, como en el caso de la inscripción de Mérida, a que - $d$ - es la consonante etimológica.

Así pues, a pesar de las dificultades y ambigüedades, valorando en su conjunto todos los indicios, parece que apuntan a una forma originaria Adaecina, que, de hecho, tenemos ahora atestiguada en Alcuéscar. Esto tiene repercusiones muy directas para la etimología del teónimo, pues automáticamente queda excluida la posibilidad de defender la etimología propuesta por D'Arbois de Juvainville.

Resta, pues, examinar la posible relación con a.irl. adaig que aparece como bastante probable, aunque no en los términos en que habitualmente se había planteado ${ }^{31}$. La secuencia inicial $a d$ - es común al teónimo hispano y al nombre irlandés, mientras que la ambigüedad de la segunda consonante del nombre irlandés, que, como vimos más arriba, podría proceder tanto de $* k$ como de $* k^{w}$ queda resuelta gracias al teónimo hispano, cuya segunda consonante sólo puede proceder de $* k$, ya que $* k^{w}$ se habría conservado, según muestra la antroponimia de la zona vetona, donde tenemos formaciones como Erguena (Yecla de Yeltes SA) y Equaesus (Yecla de Yeltes SA, Ávila). Por otra parte, la palabra irlandesa muestra que el diptongo -ae- del teónimo (que a veces aparece monoptongado en -e-, evolución fonética banal en el latín de España) en realidad ha de deberse a «in-

31 Mucho menos se puede defender que se trata de un préstamo tomado a una lengua de la península Ibérica por el irlandés, como quería Balmori 1935, pp. 220-224. 
fección» ${ }^{32}$ de la vocal - $a$ - de la segunda sílaba de la base debida a la $-i-$ del sufijo -ina, fenómeno bien atestiguado en la antroponimia de la zona vetona: Saelgius ('sal(i)k- 'sauce' + sufijo -ius), Maicira (*mak- + sufijo -ira), Caenicus (*can- + sufijo -icus), Amaenia (*aman- + sufijo -ia), Vaelcius (*walk- + sufijo -ius), etc. Y, de hecho, como señalábamos más arriba, una forma Atacina está atestiguada como antropónimo femenino en una inscripción ampuritana. Así pues, adaig y Adaecina se aclaran mutuamente.

Habría que suponer, por tanto, una forma proto-celta *adaki- que explicaría tanto el nombre irlandés como el teónimo hispano. El carácter largo originario de la - $\bar{l}$ - viene dado no sólo por la palabra irlandesa, sino también por el antropónimo femenino Ataecina, formalmente idéntico al teónimo que venimos estudiando, atestiguado en una inscripción del Nórico (CIL III 11650), para cuya -i- se ha utilizado como notación una $i$ longa. Lo mismo sucede en el caso del antropónimo Atacina de Ampurias. En cuanto a la sufijación que muestra la forma hispana frente a la irlandesa, no supone ningún problema, habida cuenta de que precisamente en las lenguas celtas, cuando se añaden sufijos en $-n$ - a polisílabos que acaban en vocal en larga, lo más frecuente es que la vocal final de la palabra sea precisamente $-\bar{l}$ (Pedersen 1913, p. 59). Por otra parte, formaciones con el sufijo -inus/-ina no faltan en la antroponimia del área vetona: Anmina (Baños de Montemayor CC), Mentina (Boada SA, Traguntía SA, Yecla de Yeltes SA), Tancinus/-a (Abertura CC, Arroyo de la Luz CC, Trujillo CC, Hinojosa del Duero, etc.).

Faltaría únicamente encontrar una etimología que resultara aceptable para dar cuenta del significado de la palabra irlandesa y del carácter que podemos suponer para la diosa hispana, pues en función del análisis realizado no resulta posible mantener ninguna de las etimologías propuestas hasta el momento para el teónimo hispano y/o el nombre irlandés.

Como mera hipótesis yo sugeriría la posibilidad de que la formación parta de una secuencia *ad-ak-, donde * $a d$ - sería el prefijo bien atestiguado en las lenguas celtas e indoeuropeas en general ${ }^{33}, \mathrm{y} * a k$ - sería una raíz bien conocida en indoeuropeo ${ }^{34}$ a partir de la cual se han derivado nume-

\footnotetext{
32 Para la interpretación de este fenómeno fonético v. Luján, e. p.

33 V. LEIA, s.u., e IEW, s.u.

34 V. Reichelt 1913, pp. 23-24, e $I E W$, s.u. La raíz está atestiguada en las lenguas celtas, p. ej. a.irl. ér 'elevado, noble', irl. med. ochar 'esquina'.
} 
rosas formaciones en las diferentes lenguas que apuntan a un significado originario 'agudo, afilado' y también 'piedra'. Precisamente a partir de este último significado quizá resultara posible explicar la forma de la que estamos tratando. Reichelt (1913) ya se ocupó de una serie de derivados de esta raíz en los que se ha producido la evolución semántica de 'piedra' a 'cielo'. Tal es el caso notablemente de a.i. aśman-, av. asan- y asman- ${ }^{35}$ y gr. $\ddot{\alpha} \kappa \mu \omega v^{36}$, cuya evolución Reichelt pudo explicar dentro de una concepción de la cúpula celeste como piedra que, además de en las tradiciones de los pueblos citados, documentó también entre los armenios y los germanos ${ }^{37}$. Curiosamente Reichelt pasó por un alto un importante testimonio celta que quizá pudiera así hallar una explicación dentro del contexto indoeuropeo: es de sobra conocida la anécdota acaecida en torno al 335 a.C. ${ }^{38}$ cuando, a la pregunta de Alejandro acerca de qué era lo que más temían, los príncipes celtas de la costa adriática habrían respondido que era que se derrumbara la cúpula celeste ${ }^{39}$. Quizá el significado 'noche' que testimonia el a.irl. adaig proceda, en relación con tales concepciones, de una especialización de significado para referirse al 'cielo nocturno'. De todas formas, no concedo a este razonamiento más valor que el de hipótesis.

Para finalizar conviene señalar las implicaciones que, de ser correcta la relación que he defendido entre Adaecina y a.irl. adaig, ésta tendría para la dialectología celta. Las hablas hispano-celtas ${ }^{40} \mathrm{y}$, entre ellas más concretamente el celtibérico, que es la que se emplea habitualmente como referencia comparativa dado que es la única de la que disponemos de información relativamente abundante, suelen ser caracterizadas como arcaizantes dentro del conjunto de las lenguas celtas, ya que, frente a las inno-

\footnotetext{
35 Sobre las que v. Mayrhofer 1987, s.u.

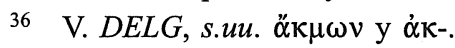

37 Tratamientos más recientes de la cuestión que matizan la propuesta de Reichelt en direcciones distintas pueden encontrarse notablemente en los trabajos de Maher 1973 y Crevatin 1974, 1975, 1976-77.

38 Ptol.Lag. en Str. VII 3.8. Véase también Arr., An. I 4.6-8 (= Ptol.Lag.2).

39 Pueden verse tratamientos recientes sobre este testimonio en los libros de Brunaux 1996, pp. 44-45, y Birkhan 1997, pp. 782-785.

40 Utilizo el término "hispano-celta" en el sentido propuesto por de Hoz 1988, como referencia a cualquier rasgo lingüístico celta presente en la península Ibérica y que hay que diferenciar de "celtibérico", que no sería sino el dialecto hispano-celta que mejor conocemos.
} 
vaciones comunes del grupo galo-britónico, las isoglosas que el celtibérico comparte con el lepóntico y el goidélico, como, respectivamente, el orden de palabras SVO o el mantenimiento de la conjunción $* k^{w} e$, se deben a conservación de arcaísmos ${ }^{41}$. En cambio, la relación entre Adaecina y adaig 'noche' supondría que tenemos una innovación léxica común a un dialecto hispano-celta y al irlandés, pues en este caso sería el grupo galo-britónico el que presentaría un arcaísmo al haber conservado la vieja palabra indoeuropea para la 'noche' ${ }^{*} n o k^{w} t$-: galés nos, bretón noz, galo trinux/trino en el calendario de Coligny.

\section{BIBLIOGRAFÍA}

Abascal Palazón, J. M., 1994: Los nombres personales en las inscripciones latinas de Hispania, Murcia.

- 1995: «Las inscripciones latinas de Santa Lucía del Trampal (Alcuéscar, Cáceres) y el culto de Ataecina en Hispania», AEspA 68, pp. 31-105.

Albertos, M. ${ }^{a}$ L., 1966: La onomástica personal primitiva de Hispania (Tarraconense y Bética), Salamanca.

Almagro, M., 1952: Inscripciones ampuritanas griegas, ibéricas y latinas, Barcelona.

D’Arbois de Juvainville, H., 1893: «Les celtes en Espagne (I)», Revue Celtique 14, pp. 357-395.

- 1906: Reseña de Leite (1905), Revue Celtique 27: 105-106.

Balmori, C. H., 1935: «Ataecina, Adaegina», Emerita 3, pp. 214-224.

Bendala, M., 1981: «Las religiones mistéricas en la España romana», en: La religión romana en Hispania, Madrid, pp. 283-299.

Binchy, D. A. (ed.), 1978: Corpus Iuris Hibernici, 6 vols., Dublín.

Birkhan, H., 1997: Kelten (Versuch einer Gesamtdarstellung ihrer Kultur), Viena.

Blázquez, J. M. ${ }^{\text {a }, ~ 1962: ~ L a s ~ r e l i g i o n e s ~ p r i m i t i v a s ~ d e ~ H i s p a n i a ~ I . ~ F u e n t e s ~ l i t e r a r i a s ~ y ~ e p i-~}$ gráficas, Roma.

- 1975: Diccionario de las religiones prerromanas, Madrid.

Brunaux, J.-L., 1996: Les religions gauloises, París.

Callejo Serrano, C., 1970: «Nuevo repertorio epigráfico de la provincia de Cáceres», AEspA 43, pp. 132-168.

41 Sobre la dialectalización de las lenguas celtas pueden verse diversos trabajos de Schmidt (p. ej., Schmidt 1986, p. 202, y 1993, pp. 71-75), así como el libro de Lambert 1994, pp. 14-19, para las relaciones entre el galo y las lenguas britónicas. Para las características arcaizantes del celtibérico puede consultarse el artículo de Gorrochategui 1994 y para una revisión de todos los rasgos que pueden utilizarse en la clasificación dialectal del celtibérico, el de de Hoz 1992, pp. 12-19. Naturalmente, algunas de las ideas expuestas en estos trabajos han de replantearse en función de la revisión de la gramática celtibérica llevada a cabo por Villar 1995. 
Cortés, S., Ocaña, E., Fernández, F. J., y Estevan, J., 1982: «Nuevas inscripciones romanas del Museo de Santa Cruz de Toledo», Museos 3, pp. 73-85.

Crevatin, F., 1974, 1975, 1976-77: «Un problema di 'antichità' indeuropee: il 'cuneo del fulmine'», Incontri Linguistici 1: 61-81; 2: 47-60; 3: 29-40.

$D E L G=$ Pierre Chantraine, Dictionnaire Étymologique de la Langue Grecque, París, 1968 etc.

$D I L=$ Royal Irish Academy, Dictionary of the Irish Language, Dublín, $1990^{2}$.

D’Encarnaçâo, J., 1975: Divindades indígenas sob o dominio romano em Portugal, Lisboa.

- 1984: Inscriçôes romanas do conventus pacensis, Coimbra.

Esteban Ortega, J., 1984: «Epígrafe sobre la diosa Ataecina», en: Actas de las Segundas Jornadas de Metodología y Didáctica de la Historia (Hist. Ant.), Cáceres, pp. 21-25.

Evans, D. E., Gaulish Personal Names, Oxford.

- 1993: «The identification of Continental Celtic with special reference to Hispano-Celtic», en: J. Untermann y F. Villar (edd.), Lengua y cultura en la Hispania prerromana, Salamanca, pp. 563-608.

García y Bellido, A., 1967: Les réligions orientales dans l'Espagne romaine, Leiden.

García-Bellido, M. ${ }^{\mathrm{a}}$ P., 1991: «Las religiones orientales en la península Ibérica: Documentos numismáticos, I», AEspA 64, pp. 37-81.

García Iglesias, L., 1972-73: Epigrafia romana de Augusta Emerita, Madrid (tesis doctoral de la Universidad Complutense).

González-Conde, M. ${ }^{a}$ P., 1986: «Elementos para una delimitación entre vettones y carpetanos en la provincia de Toledo», Lucentum 5, pp. 87-93.

— 1988: «Bassus Turobrigensis y la inscripción de Ataecina en Caleruela (Toledo)», STH (Historia Antigua) 6, pp. 131-132.

Gorrochategui, J., 1994: «El celtibérico, dialecto arcaico celta», Emerita 62, pp. 297-324.

Greene, D., 1973: «The growth of palatalization in Irish», TPhS 1973, pp. 127-136.

Ho. = Holder, A., 1896-1907: Altceltisches Sprachschatz, 3 vols., Leipzig.

de Hoz, J., 1988: «Hispano-Celtic and Celtiberian», en: G.W. MacLennan (ed.), Proceedings of the First North American Congress of Celtic Studies, Ottawa, pp. 191-207.

- 1992: "The Celts of the Iberian Peninsula», ZCP 45, pp. 1-37.

Hurtado de San Antonio, R., 1977: Corpus provincial de inscripciones latinas de Cáceres, Cáceres.

IEW = Pokorny, J., Indogermanisches Etymologisches Wörterbuch, Berna y Munich, 1949. Lambert, P.-Y., 1994: La langue gauloise, París.

Laws $=$ Ancient Laws of Ireland, Dublín 1865-1901.

LEIA = Lexique Étymologique de l'Irlandais Ancien, de J. Vendryès, continuado por E. Bachellery y P.-Y. Lambert, vol. A, Dublín, 1959; vol. B, París, 1981; vol. D, París, 1996; vol. M-N-O-P, París, 1960; vol. R-S, París, 1974; vol. T-U, París, 1978.

Leite de Vasconcelos, J., 1905: Religiôes da Lusitania, vol. 2, Lisboa.

Lewis, H. y Pedersen, H., 1989: A Concise Comparative Celtic Grammar, Gotinga [3. ${ }^{\mathrm{a}}$ edic.].

López Melero, R., 1986: «Nueva evidencia sobre el culto de Ategina: el epígrafe de Bienvenida», en: Primeras Jornadas sobre Manifestaciones Religiosas en la Lusitania (marzo de 1984), Cáceres, pp. 93-112.

Luján, Eugenio R., e. p.: «L'onomastique des Vettons: analyse linguistique», en: P.-Y. Lambert y G.-J. Pinault (eds.), Gaulois et celtique continental, París. 
Madruga Flores, J. V. y J. Salas Martín, 1995: «A propósito de teónimos indígenas en el conventus Emeritensis», Espacio, Tiempo y Forma, Serie II, H. ${ }^{a}$ Antigua 8: 331-355.

Maher, J. P., 1973: «* $\mathrm{H}_{\mathrm{a}}$ emon: '(stone) axe' and 'sky' in I-E/Battle-Axe Culture», JIES 1: 441-462.

Mayrhofer, M., 1987: Etymologisches Wörterbuch des Altindoarischen, vol. I 2, Heidelberg. Michelena, L., 1961: Reseña de Blázquez (1962), Zephyrus 12: 197-202.

$O L D=$ P. G. W. Glare (ed.), Oxford Latin Dictionary, Oxford 1985.

Pedersen, H., 1913: Vergleichende Grammatik der keltischen Sprachen, vol. 2, Gotinga.

- 1933: «Une étymologie balto-celtique», Studi Baltici 3: 69-72.

Roldán Hervás, J. M., 1968-69: «Fuentes antiguas para el estudio de los Vettones», Zephyrus 19-20: 73-106.

Roso de Luna, M., 1904: «Nuevas inscripciones romanas de la región norbense», BRAH 44: 113-137.

Salinas de Frías, M., 1986: La organización tribal de los vettones, Salamanca.

Sánchez Moreno, E., 1995: «El origen de los vetones en la historiografía española del siglo XX. ¿Implantación o formación?», Espacio, Tiempo y Forma, Serie II, H. ${ }^{a}$ Ant. 8: 475-499.

Sayas Abengoechea, J. J. y López Melero, R., 1991: «Vettones», en: J. M. ${ }^{\text {a Solana Sainz }}$ (ed.), Las entidades étnicas de la Meseta Norte de Hispania en época prerromana (Anejos de Hispania Antiqua), Valladolid, pp. 74-123.

Schmidt, K. H., 1957: «Die Komposition in gallischen Personenamen», ZCP 26: 31-301.

- 1986: «The Celtic languages in their European context», en: D. E. Evans, J. G. Griffith y E. M. Jope (edd.), Proceedings of the Seventh International Congress of Celtic Studies, Oxford.

- 1993: «Insular Celtic: P and Q Celtic», en: M. J. Ball y J. Fife (edd.), The Celtic Languages, Londres-Nueva York, pp. 64-98.

Steuding, H., 1884-86: «Ataecina, Adaegina» en: Roscher, W. H. (ed.), Lexicon der griechischen und römischen Mythologie, vol. 1, Leipzig, col. 663.

Stokes, W., 1887: «Irish femenine stems in $\hat{\imath}$ and $u$, and neuter stems in $s », K Z 28: 289-294$.

- 1894: Urkeltische Sprachschatz, Gotinga.

Thurneysen, R., 1887: «Irisches», KZ 28: 145-154.

- 1927: «Aus dem Irischen Recht IV», ZCP 16: 167-230.

- 1946: A Grammar of Old Irish, Dublín.

Tovar, A., 1949: Estudios sobre las primitivas lenguas hispánicas, Buenos Aires.

Untermann, J., 1987: «Lusitanisch, Keltiberisch, Keltisch», en: J. Gorrochategui, J. L. Melena y J. Santos (edd.), Actas del IV Coloquio sobre Lenguas y Culturas Paleohispánicas (= Veleia 2-3), Vitoria, pp. 57-76.

Urbina, D., 1993-94: «Ataecina y Vrilouco dos divinidades indígenas en Talavera de la Reina», Minius 2-3: 29-51.

de la Vega Jimeno, Miguel, 1993: «Aspectos religiosos en Talavera de la Reina y su tierra en época romana», en: Actas de las Primeras Jornadas de Arqueología de Talavera de la Reina y sus tierras, Toledo, pp. 335-348.

Villar, F., 1995: Estudios de celtibérico y de toponimia prerromana, Salamanca.

Zucca, R., e. p.: «Divinità dei Corsi e dei Sardi e loro continuazione nel pantheon romano locale», en: II Colóquio Internacional de Epigrafia (Divindades Indígenas e Interpretatio Romana), Sintra 16-18 março de 1995. 